\title{
Exposé sur l'eau au Québec
}

\author{
Water in Québec
}

\author{
par Jean-Maurice Latulippe \\ Directeur des politiques du secteur municipal \\ Ministère de l'Environnement du Québec
}

Québec water resources are, overall, largely sufficient. We have 4500 rivers and 500000 lakes, of which 30 have an area of more than $250 \mathrm{~km}^{2}$. Most of Québec's population lives on the St. Lawrence plain. Therefore, surface water flowing in this area is subject to the greatest pressures. In 1978, only $2 \%$ of municipalities wastewaters were treated. But, over the past 20 years, the health of Québec's waterways has improved markedly, as a result of a major investment program (7 billion \$) such as the Québec water purification program (PAEQ). However, there is still much to be done, especially regarding the non-point pollution, much of which resulting from farming operations.

In other respects, Québec is rethinking it's role, in order to increase the efficiency of public and municipal services. Water administration in Quebec is very fragmented and there is not much coordination or integration of the various jurisdictions between the federal, provincial and municipal levels. But, in 1992, the Québec Government initiated a watershed management approach by creating the Comite de bassin de la rivière Chaudière (COBARIC). This committee, formed with stakeholders with vested interest in the issue, submitted a report on March 26th, 1996. Based on eight main principles for an integrated approach of watershed management, the committee recommended the Minister of Environment and Wildlife to develop a master plan respecting water and to propose a financing strategy to make users liable for the use or deterioration of water. To ensure that Quebeckers endorse the objectives of the water management reform, the committee would undertake a process of information and sensibilization of the water management reform implications.

The government decided to follow the recommendations in creating the COBARIC II in April 1997 with the mandate of experimenting the proposed approach. In particular, the committee will have to elaborate a master plan respecting water. This plan means the realization of watershed management in the field. It will allow to prioritize actions and projects hierarchically, on a coherent territory (the basin), while giving the way for dialogue between users and partners. This plan will be a real decision-making tool. Results are expected at the end of the COBARIC's mandate in November 1999. Finally, the Québec government has become a member of the Réseau international des organismes de bassin (international network of watershed associations) (RIOB) in March 1996.

\section{INTRODUCTION}

Partout dans le monde, l'eau est d'actualité soit parce qu'elle est devenue rare ou impropre à la consommation ou encore parce qu'elle génère des catastrophes. Depuis quelques temps au Québec, l'eau fait l'objet de nombreux débats tant au niveau du gouvernement que de la population et des médias.

La société québécoise prend de plus en plus conscience que cette ressource constitue un atout patrimonial et économique de grande valeur. Par ailleurs, nos lois donnent à chaque personne le droit d'en faire usage, sauf dans le cas de l'eau souterraine où elle appartient au propriétaire du terrain sous lequel elle se trouve. Cet état de fait cause de plus en plus de conflits d'usages.

\section{II — L'ÉTAT DE SITUATION AU QUÉBEC}

D'un point de vue très global pour l'ensemble du territoire, les ressources en eau du Québec sont largement suffisantes pour ses besoins. Voici quelques chiffres qui nous permettent de constater son importance.

\section{- 2.1 L'eau de surface}

\section{Quantité :}

Le Québec est doté de 4500 rivières et un demi-million de lacs dont 30 ont une superficie de plus de $250 \mathrm{~km}^{2}$. Le Québec compte plus de 430 bassins majeurs dont 100 ont une superficie de drainage supérieure à $4000 \mathrm{~km}^{2}$. La superficie du bassin du fleuve Saint-Laurent représente à elle seule environ le tiers de l'ensemble du territoire québécois. 
Cette eau de surface alimente $80 \%$ de la population. Le fleuve Saint-Laurent collabore pour $45 \%$ et les lacs et rivières pour $35 \%$. Elle sert à $49 \%$ pour les besoins en eau potable des municipalités, à $46 \%$ pour les activités industrielles et à $5 \%$ pour des fins minières et l'agriculture.

\section{Qualité:}

Au Québec, la majorité de la population vit dans la plaine du Saint-Laurent. Les eaux qui y circulent subissent donc la plupart des pressions. En 1978, seulement 2\% des eaux usées municipales étaient traitées ; conséquemment la qualité du fleuve et de ses affluents était plutôt médiocre dans les zones habitées.

Aujourd'hui, nous constatons que l'état de santé de ces cours d'eau s'est sensiblement amélioré au cours des 15 dernières années. Cette amélioration est largement tributaire des efforts d'assainissement des eaux usées municipales grâce à des programmes d'investissement majeurs (7 milliards \$) tel le Programme d'assainissement des eaux (PAEQ) et aussi de la réduction marquée des rejets provenant des industries, notamment ceux du secteur des pâtes et papiers et de la métallurgie. Toutefois, malgré les efforts consentis, il reste encore beaucoup à faire pour retrouver la qualité de nos cours d'eau, notamment au niveau de la pollution diffuse qui trouve son origine pour une très large part dans les activités agricoles. La pollution provenant de certaines industries qui n'ont pas complété leurs travaux d'assainissement, des ouvrages individuels (résidences isolées), des débordements des réseaux d'égout et des multiples interventions humaines tels l'urbanisation, le déboisement, l'aménagement riverain et les lieux d'élimination des déchets est également responsable d'une partie des problèmes. Soulignons également qu'un certain nombre de municipalités ne sont pas encore dotées d'un système collectif de traitement des eaux usées. On prévoit qu'en 1999, $98 \%$ des municipalités du Québec reliées à un réseau d'égouts traiteront leurs eaux usées.

\section{- 2.2 Les eaux souterraines disponibles (secteurs habités)}

\section{Quantité :}

L'eau souterraine est présente en quantité suffisante et accessible à des coûts acceptables sur la majeure partie du territoire du Québec. On estime la quantité disponible dans les régions habitées à $200 \mathrm{~km}^{3}$, soit l'équivalent du volume déversé sur 193 jours par le fleuve Saint-Laurent à la hauteur de Québec. Cette eau souterraine alimente $20 \%$ de la population répartie sur $90 \%$ du territoire habité du Québec. Elle sert à $54 \%$ à des fins d'eau potable, d'eau de source et d'eau minérale, à $39 \%$ pour la production d'aliments (piscicultures, abreuvement du bétail, irrigation des terres, traitement et préparation de fruits et légumes) et à $7 \%$ pour les usages industriels.

\section{Qualité :}

La qualité de nos eaux souterraines est généralement bonne. Toutefois, dans certaines régions, on remarque des problèmes provenant de source naturelle ou d'activités humaines telles l'épandage de sel, d'engrais ou de pesticides ou encore d'installations inappropriées (fosses septiques, réservoirs souterrains etc.).

\section{- 2.3 Les aspects administratifs}

Comme on peut le constater, le Québec est bien pourvu en eau. Néanmoins, même si la société québécoise possède des milliers de lacs et de rivières et de l'eau souterraine en quantité et en qualité suffisantes, la répartition de l'eau douce n'est pas égale sur tout le territoire. Certains problèmes localisés nous obligent à considérer notre façon de l'exploiter. En parallèle, l'État est à redéfinir son rôle en vue d'une plus grande efficacité des services publics, des autorités municipales et des entreprises. Actuellement, la législation est fragmentée, sectorielle et partagée entre le gouvernement fédéral, le gouvernement du Québec et les municipalités. Ainsi, cinq ministères du gouvernement du Québec gèrent divers aspects de la ressource eau. À cela, il faut ajouter quelque 1400 municipalités locales, 96 municipalités régionales de comté et trois communautés urbaines de même que pas moins de huit ministères fédéraux.

Actuellement, chacun de ces organismes publics oriente son action en fonction de sa juridiction en tenant compte, non pas de l'eau, mais du mandat pour lequel on l'a créé. En conséquence, il n'y a que très peu d'intégration, que ce soit au niveau administratif, légal ou géographique, ce qui conduit à des chevauchements et des investissements de deniers publics qui ne permettent pas toujours d'atteindre les objectifs visés.

\subsection{Les faits récents}

Plusieurs événements sont survenus dans les derniers mois. Rappelons les fameuses inondations au Saguenay en juillet 1996. Vinrent ensuite des projets de privatisation des infrastructures municipales d'aqueducs et d'égout à Montréal. Un projet d'implantation d'une usine de captage et d'embouteillage d'eau souterraine dans la localité de Franklin, en Montérégie, a aussi suscité plusieurs discussions tant au niveau local que provincial. Finalement, des projets d'exportation vers les pays moins bien nantis en eau font également partie des débats de façon régulière.

Ces événements et les préoccupations grandissantes des citoyens relativement aux problématiques de l'eau, ont incité le gouvernement du Québec à poser des gestes concrets.

Ainsi, suite aux inondations du Saguenay, il a créé une commission scientifique et technique sur la gestion des barrages, la Commission Nicolet. Sur la recommandation du rapport produit par cette commission en janvier 1997, le gouvernement a déposé le 17 juin 1998 un avant-projet de loi sur la sécurité des ouvrages de retenue.

Le gouvernement a aussi annoncé qu'il entreprenait les démarches devant conduire à l'adoption d'une Politique de l'eau.

\subsection{Symposium sur l'eau}

L'approche retenue comprend plusieurs étapes dont la première a été la tenue d'un Symposium sur la gestion de l'eau au Québec en décembre 1997. Au préalable, le gouvernement avait publié un document de référence présentant des informations importantes sur l'eau du Québec, son état, sa gestion, son encadrement légal. Les objectifs de ce Symposium étaient d'actualiser l'état de nos connaissances dans le domaine de l'eau et de faire le point sur diverses problématiques.

Ce Symposium a été un véritable succès avec la présence d'environ 500 personnes et de plus d'une quarantaine de conférenciers de marque dont plusieurs de niveau international. Il a donné lieu à des discussions d'intérêt réunissant des intervenants provenant autant des municipalités que des entreprises et des centres de recherche.

Plusieurs enseignements ont pu être tirés de ce symposium. On peut mentionner les suivants : 
- Les usages de l'eau augmentent de même que les besoins exprimés par la population eu égard à cette ressource. De plus en plus de projets relatifs à l'eau voient le jour et des choix s'imposent pour assurer un partage équitable de la ressource.

- La question de la propriété de l'eau a pris de l'importance nous incitant à questionner l'encadrement juridique existant.

- L'état de santé de nos cours d'eau s'est sensiblement amélioré au cours des quinze dernières années, mais beaucoup reste à faire, particulièrement en ce qui concerne la question agricole.

- La problématique des eaux souterraines est particulière. À cet égard, il apparaît de plus en plus nécessaire de développer nos connaissances, notamment au niveau de sa quantité, sa disponibilité et sa localisation afin d'avoir une vision globale de cette problématique et d'y apporter des solutions durables.

- - Nos modes de gestion des eaux font appel à un nombre important d'intervenants. Les municipalités et les gouvernements (fédéral et du Québec) se partagent les juridictions dans plusieurs domaines, notamment la dépollution, l'aménagement du territoire, l'entretien des cours d'eau et la vitalité du secteur récréo-touristique.

- Nous ne prenons pas suffisamment conscience de la valeur de l'eau. Des mesures devraient être prises pour sensibiliser la population aux véritables coûts de son utilisation.

- L'approche de gestion des eaux par bassin versant suscite beaucoup d'intérêt et constitue peut-être une voie prometteuse pour le Québec.

En résumé, le Symposium nous a porté à nous questionner sur ce que nous voulons faire collectivement avec notre richesse patrimoniale, comment nous voulons la gérer et à quelles fins si nous voulons léguer aux générations futures un environnement de qualité. Le Symposium nous a donné l'occasion d'établir une base commune à partir de laquelle nous pourrons échanger avec l'ensemble de la population lors d'une vaste consultation publique sur la gestion de l'eau que le gouvernement a décidé de tenir pour donner suite à ce Symposium.

\subsection{Consultation publique}

Cette consultation publique débutera le 15 mars 1999. Cette prochaine étape conduisant à une future Politique de I'eau nous permettra de recueillir les différents points de vue de ceux et celles qui, dans les régions du Québec, s'intéressent de près à la préservation et à la mise en valeur de l'eau.

Cette consultation se fera sur la base d'un document de réflexion qui s'inspire entre autres des actes du Symposium. $\mathrm{Ce}$ document propose à la population certains thèmes qui devraient être retenus lors de l'élaboration de la future Politique de l'eau. Elle sera menée dans toutes les régions du Québec par le Bureau d'audiences publiques en environnement (BAPE) du Québec.

Nous pouvons d'ores et déjà avancer que la gestion des eaux souterraines, la qualité des eaux de surface, la propriété des infrastructures municipales et certains aspects économiques, telle l'exportation de l'eau, seront des sujets d'intérêt lors de la consultation publique.

La pérennité de la ressource eau et la conciliation des usages seront évidemment deux piliers indispensables au projet de Politique. Ces deux éléments sont primordiaux si nous voulons conserver une approche de développement durable.

Mentionnons à cet égard qu'en avril dernier, dans son discours sur le budget 1998-1999, le ministre des Finances a énoncé clairement que "le développement économique doit privilégier une gestion responsable des ressources naturelles et du territoire ". Le gouvernement québécois est d'avis que le respect de la qualité du milieu et de la pérennité des ressources est non seulement la garantie d'un développement durable mais peut également devenir " un appui direct à la création d'emploi ". Notre défi collectif sera donc de préserver et de mettre en valeur une richesse vitale tout en favorisant son usage de façon harmonieuse sur le territoire du Québec.

\section{III — GESTION PAR BASSIN VERSANT}

Un des points majeurs de discussions, lors du Symposium tenu en décembre 1997, a été qu'une approche de gestion des eaux par bassin versant constitue peut-être une voie prometteuse pour le Québec. En prenant connaissance des politiques et des pratiques de nombreux pays à travers le monde, on s'est aperçu que nombre de ceux-ci ont adopté ce type d'approche qui présente des avantages indéniables par rapport à la situation vécue au Québec.

Les réflexions du Québec sur le sujet ne datent pas d'aujourd'hui. Depuis plus de 25 ans, diverses expériences ont été tentées au Québec pour procéder à une gestion intégrée de l'eau, en particulier par une approche par bassin versant. Les résultats demeurent cependant très en dessous des objectifs de départ. Les causes possibles de ces insuccès sont multiples : l'absence des usagers dans le processus de gestion, l'approche très sectorielle des problématiques, la multiplicité des lois, règlements et politiques touchant l'eau, l'absence de coûts " visibles " de l'utilisation de l'eau, etc. Toutefois, certaines démarches commencent à se développer de façon intéressante.

\section{- 3.1 Historique de COBARIC I}

En avril 1992, le ministère de l'Environnement a confié à I'Association Québécoise des Techniques de l'Eau (AQTE) un mandat pour développer les caractéristiques d'un modèle de gestion de l'eau par bassin versant. Il a alors été décidé d'expérimenter cette approche sur un bassin versant, soit celui de la Chaudière. Cette rivière qui fait $6682 \mathrm{~km}^{2}$ a été retenue à cause de la diversité des usages qu'on y rencontre et également parce qu'un intérêt très clair a été manifesté par les intervenants locaux de la région Chaudière-Appalaches et d'une partie de la région de l'Estrie, qu'englobent le bassin de la rivière Chaudière. Le COBARIC I (Comité de bassin de la rivière Chaudière) a alors été créé.

Ce Comité, constitué des gens du milieu, a déposé un rapport le 26 mars 1996 dans lequel il recommande au ministre de l'Environnement, en s'appuyant sur huit principes directeurs pour une approche de gestion intégrée par bassin versant, de réaliser un schéma directeur de l'eau en concertation avec les gestionnaires et les usagers du bassin versant, de proposer une stratégie de financement qui verra à rendre redevables les utilisateurs de l'eau quant à son utilisation ou à sa détérioration et de consulter formellement la population avant leur adoption. Le COBARIC demande également que les ministères concernés soutiennent techniquement et financièrement les travaux pour en assurer le succès.

Le COBARIC I recommandait aussi que soit mise sur 
pied une interface gouvernementale qui aurait comme mandat principal de statuer sur un mode de gestion de l'eau par bassin versant sur l'ensemble du territoire habité du Québec dans une perspective de gestion intégrée du patrimoine hydrique. Le comité s'assurerait, par une démarche d'information et de sensibilisation publique, que les objectifs de cette réforme dans la gestion des eaux au Québec soient bien compris et acceptés.

Pour sa part, le gouvernement du Québec signait le 21 mars 1996 à Mexico son adhésion à la charte du Réseau international des organismes de bassin (RIOB) concrétisant son engagement à adopter ce mode de gestion des eaux par bassin versant.

\section{- 3.2 Poursuite de l'expérience COBARIC I}

De plus, le 18 avril 1997, il signifiait au ministère de l'Environnement qu'il y avait lieu de poursuivre le projet pilote portant sur la gestion intégrée de l'eau du bassin de la rivière Chaudière.

Le ministère a alors décidé d'expérimenter certains éléments du modèle proposé dans le rapport de COBARIC I, notamment l'élaboration d'un schéma directeur de l'eau, la proposition d'un modèle de financement pour la gestion intégrée de l'eau par bassin versant et la consultation de la population. Pour mener cette expérimentation, le ministère de l'Environnement créait le COBARIC II, corporation dûment constituée en vertu de la Loi sur les compagnies. Le conseil d'administration est formé de 23 membres provenant du milieu, dont notamment 8 représentants du secteur municipal, 4 représentants du secteur agricole dont 1 du secteur forestier, 4 représentants du secteur industriel, deux représentants du développement régional, un représentant du secteur énergétique et 4 membres cooptés dont un représentant du volet juridique, un représentant du secteur de la santé, un représentant du volet environnement, et un hydrogéologue.

Une entente spécifique qui a pour but d'associer et d'assurer le support technique et financier de chacun des participants à cette expérimentation, a été signée, le 24 novembre 1997, entre les intervenants régionaux représentés par les conseils de développement régional, le COBARIC II et le gouvernement représenté par le ministre de l'Environnement, le ministre responsable du Développement des régions et la ministre responsable de la région Chaudière-Appalaches.

\section{- 3.3 Schéma directeur de l'eau (SDE)}

Le principal mandat de COBARIC II consiste à élaborer un schéma directeur de l'eau. Ce dernier représente la concrétisation sur le terrain de la gestion par bassin versant. Il permettra d'orienter et de hiérarchiser les actions et les projets, sur un territoire cohérent, tout en permettant la concertation avec tous les usagers et partenaires concernés. Il sera un véritable outil décisionnel.

Ce schéma directeur de l'eau sera conçu de façon à servir pour la planification et la coordination des activités reliées à l'eau et à responsabiliser les usagers. Il servira de cadre de référence pour évaluer la compatibilité de divers projets susceptibles d'avoir des impacts sur le milieu hydrique et pour hiérarchiser et prioriser les interventions prévues sur le bassin.

Celui-ci devra tenir compte du principe de faisabilité d'une gestion concertée résultant de l'existence ou non de nos structures locales, des découpages administratifs, de l'identité culturelle et économique du secteur en s'attachant à rechercher une taille opérationnelle.

\section{Proposition de contenu du schéma directeur de l'eau}

Il appartiendra à COBARIC II de définir plus précisément le contenu du futur schéma directeur de l'eau. A titre indicatif, son élaboration devrait comprendre deux étapes.

a). État des connaissances :

La première étape consistera à faire le portrait du bassin, son hydrographie, sa topographie, les dépôts de surface, l'occupation du sol, le paysage, etc., à dresser le bilan de l'état de santé des eaux de surface et souterraines (types de pollution : organique, chimique, bactériologique, esthétique, toxique etc.), de l'état du régime hydrologique (inondations, étiages, crues), de l'état des écosystèmes aquatiques (faune et flore), de l'état physique du cours d'eau (remblayage, empiétement, creusage et redressement, prélèvement de gravier etc.) et de l'état des pressions sur la santé publique dues à la contamination de l'eau. On devra également inventorier les usages (eau potable, production hydroélectrique, irrigation, récréation, ouvrage de contrôle, villégiature, pêche, etc.) ainsi que les projets, programmes et politiques susceptibles d'avoir un impact significatif sur l'eau.

La plupart des données nécessaires à la réalisation des bilans existent déjà. Le ministère de l'Environnement a un programme complet d'inventaires et d'évaluation des cours d'eau qui pourrait avantageusement servir à préparer ces bilans. D'autres ministères, tel le ministère de l'Agriculture, des Pêcheries et de l'Alimentation, possèdent aussi beaucoup d'informations utiles.

L'ensemble de ces données servira à établir un diagnostic précis de l'état du bassin versant. De la qualité de ce diagnostic dépend en très grande partie la détermination juste et précise des enjeux et des objectifs.

b). Confection du schéma directeur :

La deuxième étape du schéma directeur de l'eau consistera, à partir du diagnostic de l'état du bassin, à formuler des observations et des hypothèses en vue de déterminer les enjeux et les objectifs en termes de milieu, de protection, de réhabilitation des écosystèmes aquatiques, de préservation des milieux humides, etc. et d'usage que l'on veut faire du cours d'eau (utilisation et partage de la ressource, satisfaction des usagers, valorisation de la ressource).

En fonction des objectifs à atteindre, le schéma directeur de l'eau contiendra un plan d'actions prioritaires qui visera une hiérarchie de l'ensemble des interventions d'assainissement, de prévention, de restauration et de mise en valeur des cours d'eau du bassin versant. Ces mesures s'inscriront dans la perspective d'une satisfaction optimale de tous les usagers du bassin tout en assurant également le maintien et la préservation des écosystèmes.

Le schéma directeur de l'eau, qui sera développé par COBARIC II, servira de prototype permettant la coordination des actions liées à l'eau à l'échelle d'un bassin versant tout en organisant la responsabilisation des usagers. Il est important de mentionner toutefois que ce schéma n'aura pas force exécutoire à moins qu'une décision gouvernementale en précise les modalités d'application.

\subsection{Stratégie de financement pour la gestion intégrée de l'eau}

Le deuxième mandat de COBARIC II, en plus de confectionner un schéma directeur de l'eau, consiste à proposer un cadre législatif, financier et opérationnel qui assurera l'autonomie financière pour la gestion de l'eau à l'échelle d'un bassin versant et qui permettra de rendre redevable les utili- 
sateurs de l'eau autant pour son utilisation que pour sa détérioration. Ce cadre permettra le développement de divers outils économiques pour accroître la responsabilité des utilisateurs de l'eau, réaliser des travaux ou mettre en place des équipements découlant de l'application du schéma directeur de l'eau. Le COBARIC II doit également, dans le cadre de ce mandat, proposer des mécanismes de conciliation et d'harmonisation du schéma directeur de l'eau avec les pouvoirs municipaux et les schémas d'aménagement.

\subsection{Stratégie d'information et de communication de la population}

En troisième lieu, le COBARIC II devra procéder à une consultation de la population du territoire drainé par le bassin versant de la rivière Chaudière sur le schéma directeur de l'eau et le mode de financement pour la gestion intégrée de l'eau par bassin versant.

\section{- 3.6 Résultats attendus}

Enfin, COBARIC II devra présenter au ministre de l'Environnement et de la Faune, avant le 24 novembre 1999, un rapport comprenant le schéma directeur de l'eau, la stratégie de mise en application et de financement de ce dernier de même que les résultats des consultations menées auprès de la population. Ce rapport inclura entre autres les arguments ou constatations pour ou contre le schéma directeur de l'eau et la proposition de financement pour la gestion intégrée de l'eau par bassin versant.

\section{- 3.7 Autres initiatives de gestion de l'eau}

Outre COBARIC II, de nombreux organismes de rivière sont en train de s'organiser pour mieux gérer les cours d'eau. On dénombre aujourd'hui plus d'une soixantaine de ces associations sur les principales rivières du Québec dont notamment, les rivières Saint-François, Saint-Maurice, l'Assomption, Jacques-Cartier, des Milles-Iles et SaintCharles pour n'en nommer que quelques-unes.

Une trentaine de ces associations se sont regroupées pour former "Le Réseau des organismes de rivières du Québec " (Réseau d'Or). Ce réseau permet la diffusion de l'information pertinente à la gestion de l'eau et constitue un interlocuteur provincial en matière de gestion intégrée des rivières.

\section{IV $\square$ CONCLUSION}

La gestion de l'eau constitue une des grandes priorités du Gouvernement du Québec. Les actions qu'il a posées nous démontrent l'importance qu'il accorde à cette ressource, d'abord par la création de la Commission Nicolet suite aux inondations du Saguenay, ensuite par la tenue du Symposium sur l'eau en décembre 1997 qui sera suivi d'une vaste consultation publique sous l'égide du BAPE, le tout devant conduire à une future Politique de l'eau. Cette consultation permettra aux citoyens et citoyennes du Québec, individuellement ou au nom d'une association, d'exprimer un point de vue sur la gestion de l'eau au Québec. Le rapport du BAPE sera remis au ministre de l'Environnement au plus tard le 15 mars 2000. De ce rapport et des autres éléments mis en place pour mieux connaître les paramètres d'une meilleure gestion de l'eau (COBARIC, RIOB, Symposium, etc.) une Politique québécoise de l'eau devrait voir le jour à l'aube du 3ème millénaire. 